\title{
Intrinsic spectra of H.E.S.S. blazars : what would we see without EBL absorption
}

\section{Romoli}

Dublin Institute for Advanced Studies, 31 Fitzwilliam Place, Dublin 2, Ireland

D. A. Sanchez

LAPP, Univ. de Savoie, CNRS/IN2P3, Annecy-le-Vieux F-74941, France

E-mail: david.sanchez@lapp.in2p3.fr

M. Lorentz

DSM/Irfu, CEA Saclay, F-91191 Gif-Sur-Yvette Cedex, France

P. Brun

DSM/Irfu, CEA Saclay, F-91191 Gif-Sur-Yvette Cedex, France

\section{on behalf of the H.E.S.S. collaboration}

\begin{abstract}
The vast majority of extragalactic sources detected in the very high energy $(\mathrm{E}>100 \mathrm{GeV})$ domaine are active galactic nuclei (AGN) located at cosmological distances. During their travel towards Earth, the emitted gamma-rays suffer from absorption by the extragalactic background light (EBL). The density of the EBL is not very well constrained by direct or indirect measurement which leads to uncertainties on the intrinsic spectrum of the sources. High-quality AGN spectra obtained with the High Energy Stereoscopic System (H.E.S.S.) have been used to perform a model-independent measurement of the EBL spectral energy distribution. While the precision of this measurement remains limited, it reflects the sensitivity of H.E.S.S. alone to the EBL and offers the possibility to access the intrinsic spectra of AGNs in a consistent manner, taking into account the derived uncertainties on the EBL spectral energy distribution.

In this contribution, we study the intrinsic spectra as measured by H.E.S.S. and by the Fermi Large Area Telescope (LAT) of several blazars of the H.E.S.S. sky. This provides the opportunity to have new insight into the emission processes at play in the jets of AGN. The data presented consist of monitoring data of quiescent state of blazars and also bright blazar flares, such as PKS 2155-304, Mrk 421, recorded by H.E.S.S.
\end{abstract}

35th International Cosmic Ray Conference - ICRC2017-

10-20 July, 2017

Bexco, Busan, Korea

* Speaker. 


\section{Introduction}

While traveling cosmological distances, the VHE $\gamma$-rays coming from blazars interact with lower energy photons from the extragalactic background light (EBL), resulting in an energy- and redshift-dependent absorption. The density of EBL photons is subject to uncertainties, direct measurements suffering from the contamination of foreground sources [11] (e.g. zodiacal light), and galaxy numbers counts being only considered as lower limits [10]. As a consequence, the intrinsic VHE spectra of distant sources is still unknown.

The EBL absorption leaves a clear imprint on the intrinsic source spectrum, forming the basis of the first detection of the EBL with the H.E.S.S. telescopes using bright or distant blazars [2]. This measurement, recently updated by [12], allowed to probe the intrinsic spectral properties, in the VHE range, of the sources used to derive the EBL measurement in a coherent manner.

\section{Analysis}

In this study, the same data set as in [12] is used and the local EBL density considered for the correction of the spectra is also derived in [12]. When fitting together Fermi-LAT and H.E.S.S.data, only contemporaneous Fermi data were considered with the H.E.S.S.data set. These were split into flux bins in [12], and have been merged. Faint Fermi sources have their spectra derived with 8 years of data. Time ranges of the Fermi-LAT analysis are given in Table 1.

\subsection{H.E.S.S data sets}

The High Energy Stereoscopic System (H.E.S.S.) is located in the Khomas Highlands, Namibia ( $\left.23^{\circ} 16^{\prime} 18^{\prime \prime} \mathrm{S}, 16^{\circ} 30^{\prime} 01^{\prime \prime} \mathrm{E}\right)$, at an altitude of $1800 \mathrm{~m}$ above sea level. In its first phase, H.E.S.S. was an array of four identical imaging atmospheric Cherenkov telescopes. In 2012, a fifth telescope has been added in the center of the array. This study only considers data taken during the first phase.

A large part of the H.E.S.S. observation time is devoted either to the detection of new blazars, or the monitoring of well known VHE sources or to target of opportunity pointings. The analyzed sources and the corresponding data sets have been presented in [12] together with the analysis procedure. In total, 9 sources are included in this study. Name, coordinates and redshift of the sources are given in Table 1.

Mrk 421 has been observed by H.E.S.S. in 2004 (3 data sets) and during a flaring episode in 2010 [14] (2 data sets). All the observations taken on PKS 2005-489 have been divided into two flux bins to ensure sufficient statistics. PKS 2155-304 is extensively observed by H.E.S.S. since the beginning of the experiment which recorded an exceptional flare in 2006 [6]. This event has been divided in 7 flux bins and the data taken in 2008 during a multi-wavelengh campaign [7] makes the last data set for this source.

Spectral points were extracted using a Bayesian unfolding technique $[8,3]$ allowing them to be obtained independently of any spectral model, along with as the correlation matrix between points. Points are then corrected by the EBL model as obtained in [12] and fitted taking into account the correlation matrix. The uncertainty derived on the EBL photon density is added in quadrature to the statistical errors of the H.E.S.S.data. 


\subsection{Fermi-LAT analysis}

The large area telescope (LAT) on-board the Fermi satellite is a pair conversion detector with a silicon strip tracker on the top of a calorimeter [9]. A segmented anti-coincidence shield allows the rejection of the charged particles. The bulk of LAT observations are performed in an all-sky survey mode allowing to observe all parts of the sky for about 30 minutes every 3 hours.

Data and software are publicly available from the Fermi Science Support Center (FSSC). Each source has been analyzed in the same way using the Enrico Python package [13] adapted for PASS 8 analysis. A region of interest (ROI) of $15^{\circ}$ radius, centered on the source was defined to extract the spectral parameters. The PASS 8 data (event class 128 and event type 3) were used together with the corresponding response functions P8R2_SOURCE_V6. Note that, the time range of the analysis is given in Table 1 . In addition, cut on the zenith angle $\left(<90^{\circ}\right)$ was applied to remove the Earth albedo.

The sky model has been created including all the sources of the 3FGL [5], adding the Galactic diffuse emission using the file gll_iem_v06.fits [4] and the isotropic background using iso_P8R2_SOURCE_V6_v06.txt.

\section{Derivation of the spectral parameters}

In order to derive the spectral parameters of the sources, the H.E.S.S. and Fermi data points have been fitted in log-log space. In the H.E.S.S. energy range, correlation between points (evaluated using the covariance matrix) is taken into account. For this a simple $\chi^{2}$ fit was used and data have been fitted with a power-law (LP), a log-parabola (LP) and a power-law with an exponential cut-off (PLEC) model. The best model is chosen based on the values of the $\chi^{2}$ with a cut at $5 \sigma$

The first step is to only fit the H.E.S.S.data. In this case, the vast majority of the data set is well fitted with a PL. Figure 1 show fitted spectral index as a function of the normalisation of the spectra at $1 \mathrm{TeV}$. 6 sources exhibit a low flux together with a hard spectral index $(<2.5)$ : 1ES 0229+200, 1ES 0347-121, 1ES 0414+009, 1ES 1101-232, 1ES 1312-423 and H 2356-309 (see section 3.2).

During the 2006 flare, PKS 2155-304 exhibited spectral variability [1] which is confirmed here. Important spectral variability is also found for Mkr 421 during the 2 flares of 2004 and 2010 recorded by H.E.S.S.. Interestingly the highest state in 2010 is compatible with the middle flux state in 2014 but there is not enough data to draw any conclusion. PKS 2005-489 does not seem to show spectral variability but here again, statistics are lacking for a firm conclusion.

\subsection{Constraining the peak of the Spectral Energy Distribution}

Three sources have their $\gamma$-ray SED peak constrained by the Fermi-LAT and H.E.S.S.data : Mrk 421, PKS 2005-489 (full data set) and PKS 2155-304 (2008 data set). The peak energy is in the $\mathrm{GeV}$ range (Table 2). For Mrk 421, this result is obtained during a flaring episode which is not the case of the 2 other sources. Nevertheless, the flux of all the sources is higher than the flux of the other blazars of the sample (Fig 4). 
Table 1: List of source (Name, RA, Dec and redshift) used in this study. The last column gives the time range of the Fermi-LAT analysis in MET (Mission Elapsed Time), see section 2.2.

\begin{tabular}{|l|l|l|l|l|}
\hline Name & RA & Dec & redshift & Fermi time range \\
\hline \hline 1ES 0229+200 & 023253.2 & +201621 & 0.1396 & $239557418.0-491961604.0$ \\
1ES 0347-121 & 034923.0 & -115838 & 0.188 & $239557418.0-491961604.0$ \\
1ES 0414+009 & 041652.9 & +010520 & 0.287 & $239557418.0-491961604.0$ \\
1ES 1101-232 & 110336.5 & -232945 & 0.186 & $239557418.0-491961604.0$ \\
Markarian 421 & 110419 & +381141 & 0.031 & $288057602.0-288403202.0$ \\
1ES 1312-423 & 131458.5 & -423549 & 0.105 & $239557418.0-491961604.0$ \\
PKS 2005-489 & 200927.0 & -484952 & 0.071 & $239557418.0-491961604.0$ \\
PKS 2155-304 & 215852.7 & -301318 & 0.116 & $241315201.0-242438401.0$ \\
H 2356-309 & 235909.4 & -303722 & 0.165 & $239557418.0-491961604.0$ \\
\hline
\end{tabular}

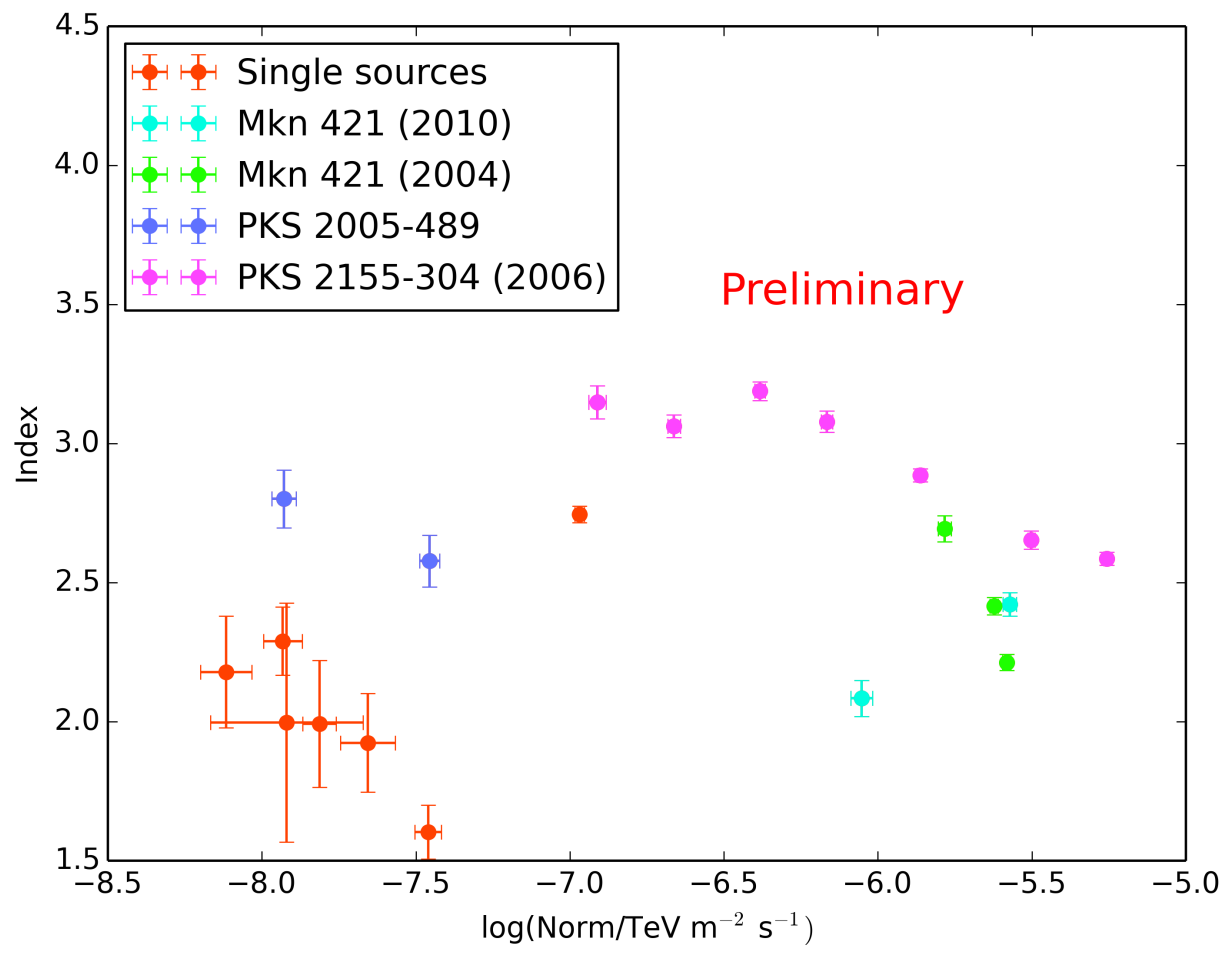

Figure 1: Results of the fit of the unfolded H.E.S.S.data points with a simple PL. The $\mathrm{X}$ axis is the normalisation at $1 \mathrm{TeV}$ and the $\mathrm{Y}$ axis is the spectral index Gamma. Red points are the results for 1ES 0229+200, 1ES 0347-121, 1ES 0414+009, 1ES 1101-232, 1ES 1312-423, PKS 2155-304 (in 2008) and H 2356-309. The bright state of Mrk 421, PKS 2005-489 and PKS 2155-304 are shown separately. 

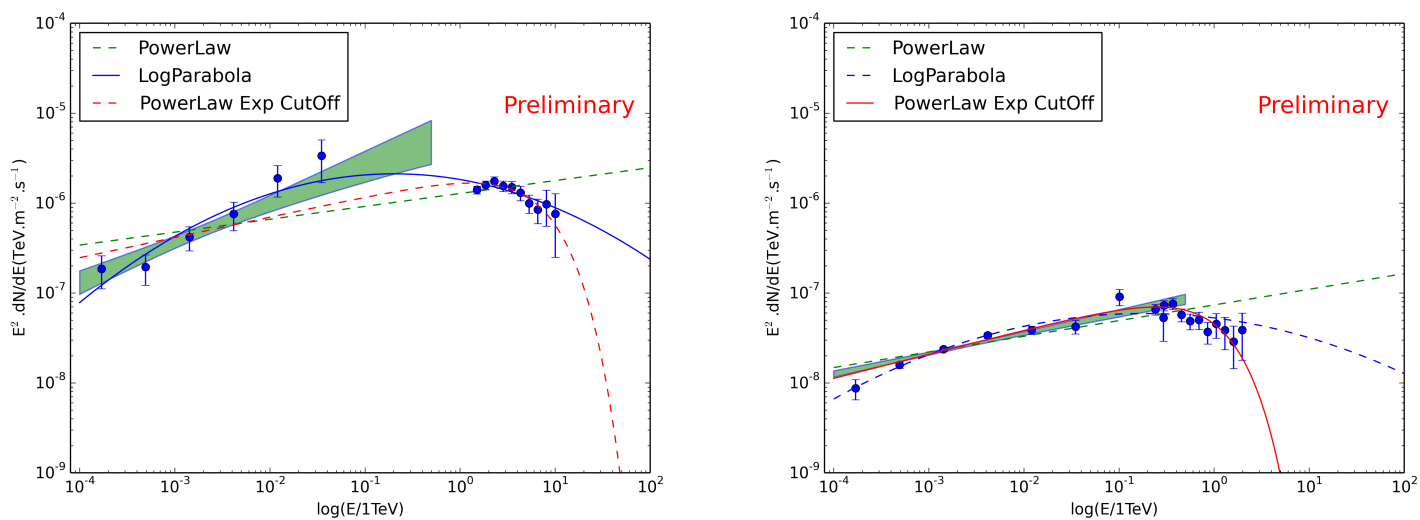

Figure 2: SED of Mrk 421 (left) and PKS 2005-489 (right). Data points are the Fermi-LAT and H.E.S.S.data. The green butterfly is the $1 \sigma$ contour of the Fermi-LAT analysis. Data were fitted with a PL, a LP and a PLEC. The best model is the one represented with a plain line (LP for Mrk 421 and PLEC for PKS 2005-489).

\subsection{Bayesian limits on the peak position}

Six sources have their $\gamma$-ray SED best fitted with a simple power-law model. It is worth noting that, the found index of the joint Fermi-LAT-H.E.S.S. fit is in good agreement with the results of the Fermi-LAT analysis (Fig. 3). This indicates a continuation of the PL in the intrinsic spectrum of each source and that the peak position $E_{\text {peak }}$ is located at high energy (at or above $100 \mathrm{GeV}$ ).

In order to constrain the value of $E_{\text {peak }}$, lower limit values have been obtained using a bayesian approach. For this, the PLEC is used as the spectral model with the collection of data points (from H.E.S.S.and Fermi) $\phi_{i}$ (measured at an energy $E_{i}$ with an uncertainties $\sigma_{i}$ ) will be used and noted $Y$.

For this procedure, we define $\Theta$ to be the parameters of our model. The used spectral model being the PLEC then, the evaluated flux at a given energy is $\Phi\left(E_{i}\right)=f\left(N, \Gamma, E_{\text {cut }}\right)$. This results that $\Theta=\left\{N, \Gamma, E_{\text {cut }}\right\}$.

Following Bayes' Theorem, it is possible to to write the posterior probability $P(\Theta \mid Y)$ as the product of the likelihood $P(Y \mid \Theta)$ and the prior probability $P(\Theta)$ :

$$
P(\Theta \mid Y) \propto P(\Theta) P(Y \mid \Theta) .
$$

The likelihood $P(Y \mid \Theta)$ can be written as

$$
\prod_{i} P\left(\phi_{i} \mid \Theta\right)=\prod_{i} \mathscr{N}\left(\phi_{i} \mid \Phi\left(E_{i}\right), \sigma_{i}\right) .
$$

For the purpose of this model, each of the parameters are assumed to be independent, such that the prior $P(\Theta)$ can be expressed as

$$
P(\Theta) \propto \mathscr{N}\left(N \mid N_{\text {Fermi }}, d N_{\text {Fermi }}\right) \mathscr{N}\left(\Gamma \mid \Gamma_{\text {Fermi }}, d \Gamma_{\text {Fermi }}\right) P\left(E_{\mathrm{c}}\right) .
$$

$N_{\text {Fermi }}$ and $\Gamma_{\text {Fermi }}$ and the associated errors are the results of the spectral fit of the Fermi-LAT data with a simple PL. Moreover, to ensure that this prior tends to zero for large value of $E_{\mathrm{c}}$, it this assumed that $P\left(E_{\mathrm{c}}\right) \propto 1 / E_{\mathrm{c}}$. 
For the computation of the limit, LAT data above $1 \mathrm{GeV}$ and H.E.S.S.data were used. Table 3 and Figure 4 give the results on position of the peak at $95 \%$ confidence level in the $v f(v)$ representation. The energy of the peak $E_{\text {peak }}$ and value of it $\left(E^{2} d N / d E_{\text {peak }}\right)$ are of course correlated since the lower limit is put on the value of the cut-off energy. One can note that for most of the sources except 1ES $0414+009$, a lower limit on the peak energy of $200 \mathrm{GeV}$ is obtained with a low flux with respect to the three sources for which the peak is constrained.
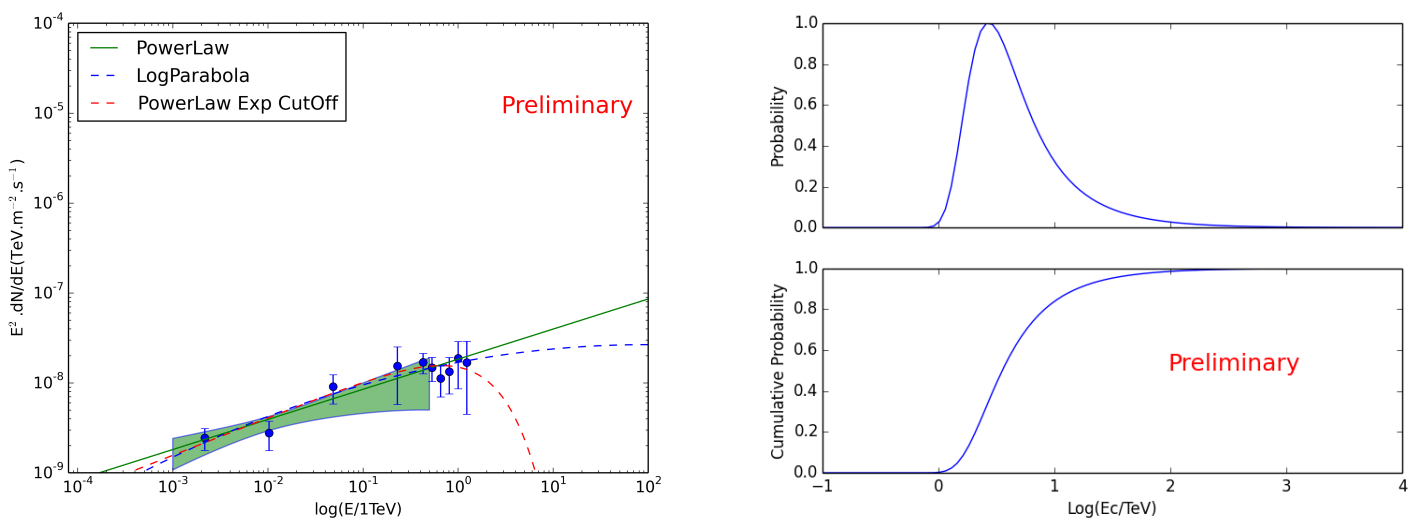

Figure 3: Left panel: SED of 1ES 0229+200, Fermi-LAT-H.E.S.S.data were fitted with 3 different models (PL, LP and PLEC). Right panel: Posterior probability and cumulative obtained for 1ES 0229+200 using the bayesian model described in this work.

\section{Summary}

H.E.S.S. and Fermi-LAT data together with the recent measurement of the EBL density by H.E.S.S. were used to study the intrisic spectra of blazars visible by H.E.S.S. This results in the following findings :

- Considering only the H.E.S.S. data, the vast majority of the H.E.S.S. spectra (fitted alone) are well represented by a PL,

- Only three sources (the three brightest sources at $\mathrm{TeV}$ energies) have their peak in the $v f(v)$ representation constrained by combined Fermi and H.E.S.S. data sets,

- For the other sources, a limit has been derived on this peak position.

\section{Acknowledgements}

The support of the Namibian authorities and of the University of Namibia in facilitating the construction and operation of H.E.S.S. is gratefully acknowledged, as is the support by the German Ministry for Education and Research (BMBF), the Max Planck Society, the German Research Foundation (DFG), the Alexander von Humboldt Foundation, the Deutsche Forschungsgemeinschaft, the French Ministry for Research, the CNRS-IN2P3 and the Astroparticle Interdisciplinary 
Table 2: Best fit model of the $\gamma$-rays data and peak position and flux value in the $v f(v)$ representation of the sources which exhibit a significant turn over in this representation.

\begin{tabular}{|l|l|l|l|}
\hline Name & Model & $\begin{array}{l}E_{\text {peak }} \\
{[\mathrm{TeV}]}\end{array}$ & $\begin{array}{l}E^{2} d N / d E_{\text {peak }} \\
10^{-7}\left[\mathrm{TeV} \cdot \mathrm{m}^{-2} \cdot \mathrm{s}^{-1}\right]\end{array}$ \\
\hline Mrk 421 & LP & $0.20 \pm 0.09$ & $21.4 \pm 2.3$ \\
PKS 2005-489 & PLEC & $0.25 \pm 0.02$ & $0.70 \pm 0.10$ \\
PKS 2155-304 & PLEC & $0.081 \pm 0.009$ & $3.5 \pm 0.7$ \\
\hline
\end{tabular}

Table 3: Lower limits at 95\% confidence level obtained on cut-off energy of the PLEC $E_{\mathrm{c}}$ with the bayesian model developed in this work. Corresponding peak position $E_{\text {peak }}$ and flux in the $v f(v)$ representation.

\begin{tabular}{|l|l|l|l|}
\hline Name & $\begin{array}{l}E_{\mathrm{c}} \\
{[\mathrm{TeV}]}\end{array}$ & $\begin{array}{l}E_{\text {peak }} \\
{[\mathrm{TeV}]}\end{array}$ & $\begin{array}{l}E^{2} d N / d E_{\text {peak }} \\
10^{-8}\left[\mathrm{TeV} . \mathrm{m}^{-2} . \mathrm{s}^{-1}\right]\end{array}$ \\
\hline 1ES 0229+200 & 1.63 & 0.52 & 1.09 \\
1ES 0347-121 & 0.81 & 0.33 & 1.45 \\
1ES 0414+009 & 0.45 & 0.062 & 0.90 \\
1ES 1101-232 & 0.45 & 0.4 & 1.72 \\
1ES 1312-423 & 1.29 & 0.22 & 0.70 \\
H 2356-309 & 1.02 & 0.25 & 1.2 \\
\hline
\end{tabular}

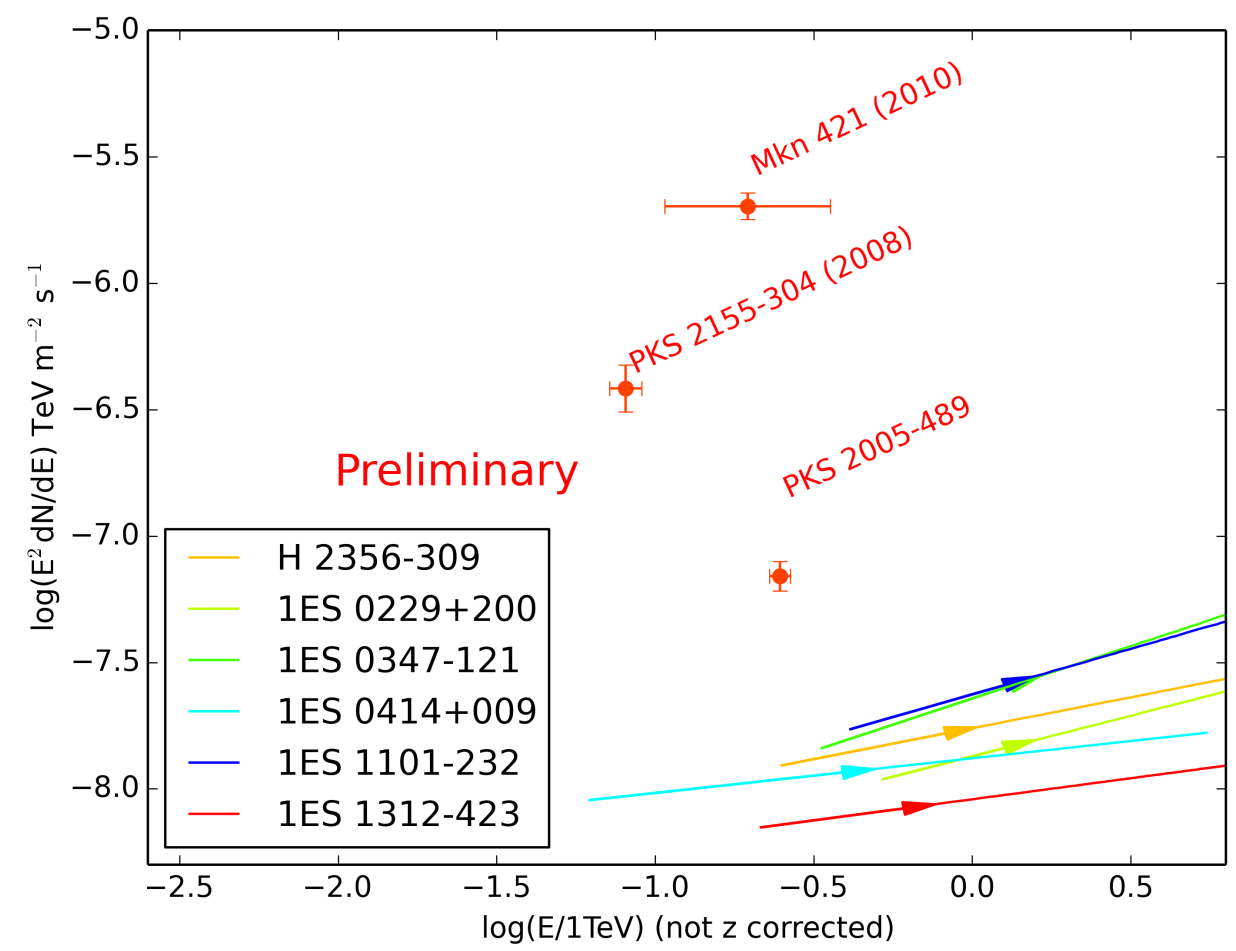

Figure 4: $\log$ of the $E_{\text {peak }}$ as a function of the value in the SED representation. Sources for which only an lower-limit can be derived, see table 3, an arrow is shown which gives the relation between the peak position and the peak value (assuming a PLEC model). 
Programme of the CNRS, the U.K. Science and Technology Facilities Council (STFC), the IPNP of the Charles University, the Czech Science Foundation, the Polish National Science Centre, the South African Department of Science and Technology and National Research Foundation, the University of Namibia, the National Commission on Research, Science \& Technology of Namibia (NCRST), the Innsbruck University, the Austrian Science Fund (FWF), and the Austrian Federal Ministry for Science, Research and Economy, the University of Adelaide and the Australian Research Council, the Japan Society for the Promotion of Science and by the University of Amsterdam. We appreciate the excellent work of the technical support staff in Berlin, Durham, Hamburg, Heidelberg, Palaiseau, Paris, Saclay, and in Namibia in the construction and operation of the equipment. This work benefited from services provided by the H.E.S.S. Virtual Organisation, supported by the national resource providers of the EGI Federation.

\section{References}

[1] H.E.S.S. Collaboration, Abramowski, A., Acero, F., et al. 2012, A\&A, 539, A149

[2] H.E.S.S. Collaboration, Abramowski, A., Acero, F., et al. 2013, A\&A, 550, A4

[3] Abramowski, A., Acero, F., Aharonian, F., et al. 2013, PRD, 88, 102003

[4] Acero, F., Ackermann, M., Ajello, M.,et al. 2016, ApJS, 223, 26

[5] Acero, F., Ackermann, M., Ajello, M., et al. 2015, ApJS, 218, 23

[6] Aharonian, F., Akhperjanian, A. G., Bazer-Bachi, A. R., et al. 2007, ApJL, 664, L71

[7] Aharonian, F., Akhperjanian, A. G., Anton, G., et al. 2009, ApJL, 696, L150

[8] Albert, J., Aliu, E., Anderhub, H., et al. 2007, Nuclear Instruments and Methods in Physics Research A, 583, 494

[9] Atwood, W. B., Abdo, A. A., Ackermann, M., et al. 2009, ApJ, 697, 1071

[10] Dole, H., Lagache, G., Puget, J.-L., et al. 2006, A\&A, 451, 417

[11] Dwek, E., \& Krennrich, F. 2013, Astroparticle Physics, 43, 112

[12] H.E.S.S. collaboration, Submitted to A\&A

[13] Sanchez, D. A. \& Deil, C. 2013, in Proceedings of the 33rd International Cosmic Ray Conference (ICRC 2013)

[14] Tluczykont, M., \& H.E.S.S. Collaboration 2010, 25th Texas Symposium on Relativistic Astrophysics, 197 